\title{
A constructive proof of the general Lovász Local Lemma
}

\author{
Robin A. Moser* \\ Institute for Theoretical Computer Science \\ Department of Computer Science \\ ETH Zürich, 8092 Zürich, Switzerland \\ robin.moser@inf.ethz.ch
}

\author{
Gábor Tardos ${ }^{\dagger}$ \\ School of Computing Science \\ Simon Fraser University \\ Burnaby, BC, Canada \\ and \\ Rényi Institute \\ Budapest, Hungary \\ tardos@cs.sfu.ca
}

May 2009

\begin{abstract}
The Lovász Local Lemma EL75 is a powerful tool to non-constructively prove the existence of combinatorial objects meeting a prescribed collection of criteria. In his breakthrough paper Bec91, Beck demonstrated that a constructive variant can be given under certain more restrictive conditions. Simplifications of his procedure and relaxations of its restrictions were subsequently exhibited in several publications Alo91, MR98, CS00, Mos06, Sri08, Mos08. In Mos09, a constructive proof was presented that works under negligible restrictions, formulated in terms of the Bounded Occurrence Satisfiability problem. In the present paper, we reformulate and improve upon these findings so as to directly apply to almost all known applications of the general Local Lemma.
\end{abstract}

Key Words and Phrases. Lovász Local Lemma, constructive proof, parallelization.

\section{Introduction}

Let $\mathcal{A}$ be a finite collection of mutually independent events in a probability space. The probability that none of these events happen is exactly $\prod_{A \in \mathcal{A}}(1-\operatorname{Pr}[A])$. In particular, this probability is positive whenever no event in $\mathcal{A}$ has probability 1. László Lovász's famous Local Lemma [EL75] allows for limited dependence among the events, but still concludes that with positive probability none of the events happen if the individual events have bounded probability. Here is the lemma in a very general form.

Theorem 1.1. EL75] Let $\mathcal{A}$ be a finite set of events in a probability space. For $A \in \mathcal{A}$ let $\Gamma(A)$ be a subset of $\mathcal{A}$ satisfying that $A$ is independent from the collection of events $\mathcal{A} \backslash(\{A\} \cup \Gamma(A))$. If there exists an assignment of reals $x: \mathcal{A} \rightarrow(0,1)$ such that

$$
\forall A \in \mathcal{A}: \operatorname{Pr}[A] \leq x(A) \prod_{B \in \Gamma(A)}(1-x(B))
$$

then the probability of avoiding all events in $\mathcal{A}$ is at least $\prod_{A \in \mathcal{A}}(1-x(A))$, in particular it is positive.

\footnotetext{
${ }^{*}$ Research is supported by the SNF Grant 200021-118001/1

${ }^{\dagger}$ Supported by NSERC grant 329527, and by OTKA grants T-046234, AT-048826 and NK-62321
} 
The original proof of this statement is non-constructive and does not yield an efficient procedure for searching the probability space for a point with the desired property. The purpose of the present paper is to give an alternative, algorithmic proof that provides such a procedure. This is not the first attempt to do so. In Bec91, Beck achieved a significant breakthrough demonstrating that algorithmic versions of the Local Lemma exist. He formulated his strategy in terms of hypergraph 2-coloring as a specific application of the lemma and proved that if in a hypergraph, every edge contains at least $k$ vertices and shares common vertices with no more than roughly $2^{k / 48}$ other edges, then a polynomial time algorithm can 2-color the vertices without producing a monochromatic edge. The existential version of the Local Lemma on the other hand allows for every edge to share vertices with roughly $2^{k} / e$ other edges and guarantees the existence of such a coloring. Subsequently, several authors have attempted to improve upon the gap between the existential version and its constructive counterparts. Alon improved the threshold to essentially $2^{k / 8}$ using a simpler and randomized variant of Beck's algorithm [Alo91. Molloy and Reed provided in [MR98 a general framework capturing the requirements a particular application has to meet so as to become tractable by the tools of Beck and Alon. A small error in this result was recently fixed in PT09. Czumaj and Scheideler's contribution in CS00 extended known algorithmic versions to somewhat more general cases where the edges of the hypergraph in question need not be of uniform size. Srinivasan in Sri08 provided another improvement that reduced the gap to a threshold of essentially $2^{k / 4}$ along with a series of other advantages over the previous approaches. In Mos08, yet another variant was presented that achieves a polynomial running time for instances up to a neighborhood size of roughly $2^{k / 2}$ and finally in Mos09, the threshold was lowered to roughly $2^{k} / 32$. In the present paper, we reformulate and improve upon the last cited result both so as to get rid of the now unnecessary constant in the hypothesis and so as to directly apply to almost all applications of the Local Lemma known so far. The only restriction we have to impose upon the general setting in the non-constructive version as formulated above will be that we consider events determined by different subsets of underlying mutually independent random variables and $\Gamma(A)$ consists of all events that depend on some of the same variables as $A$. See the exact formulation below. While this appears to be necessary in order to get any algorithmic access to the problem, it seems as well to be the case in almost all known applications.

Let $\mathcal{P}$ be a finite collection of mutually independent random variables in a fixed probability space $\Omega$. We will consider events $A$ that are determined by the values of some subset $S \subseteq \mathcal{P}$ of these variables. In such a case we say that an evaluation of the variables in $S$ violates $A$ if it makes $A$ happen. Clearly, if $A$ is determined by $\mathcal{P}$, then there is a unique minimal subset $S \subseteq \mathcal{P}$ that determines $A$. We denote this set of variables by $\operatorname{vbl}(A)$ and assume throughout the paper that this set is given to all algorithms dealing with the event $A$.

Let $\mathcal{A}$ be a finite family of events in $\Omega$ determined by $\mathcal{P}$. We define the dependency graph $G=G_{\mathcal{A}}$ for $\mathcal{A}$ to be the graph on vertex set $\mathcal{A}$ with an edge between events $A, B \in \mathcal{A}$ if $A \neq B$ but $\operatorname{vbl}(A) \cap \operatorname{vbl}(B) \neq \emptyset$. For $A \in \mathcal{A}$ we write $\Gamma(A)=\Gamma_{\mathcal{A}}(A)$ for the neighborhood of $A$ in $G$. Note that $\Gamma(A)$ satisfies the requirement in Theorem 1.1 as $A$ is determined by the variables in $\operatorname{vbl}(A)$ and the events in $\mathcal{A} \backslash(\{A\} \cup \Gamma(A))$ are determined by the rest of the variables in $\mathcal{P}$.

Given the family $\mathcal{A}$ of events as above our goal is not only to show that there exists an evaluation that does not violate any event in the family but to efficiently find such an evaluation. The algorithm we suggest (Algorithm 1.1) is as simple and natural as it can get: We start with a random point in $\Omega$ and maintain an evaluation $v_{P}$ of each variable $P \in \mathcal{P}$. We check whether some event in $\mathcal{A}$ is violated. If so, we arbitrarily pick a violated event $A \in \mathcal{A}$ and sample another random assignment of values for the variables in $\operatorname{vbl}(A)$ on which $A$ depends, each one independently and according to its distribution while not changing the values of the variables in $\mathcal{P} \backslash \operatorname{vbl}(A)$. We call this a resampling of the event $A$. We continue resampling violated events until no such event exists anymore. We will prove that this simple algorithm quickly terminates, i.e., it quickly reaches an evaluation of the variables not violating any of the events in $\mathcal{A}$ if the conditions of the Local Lemma are satisfied. 


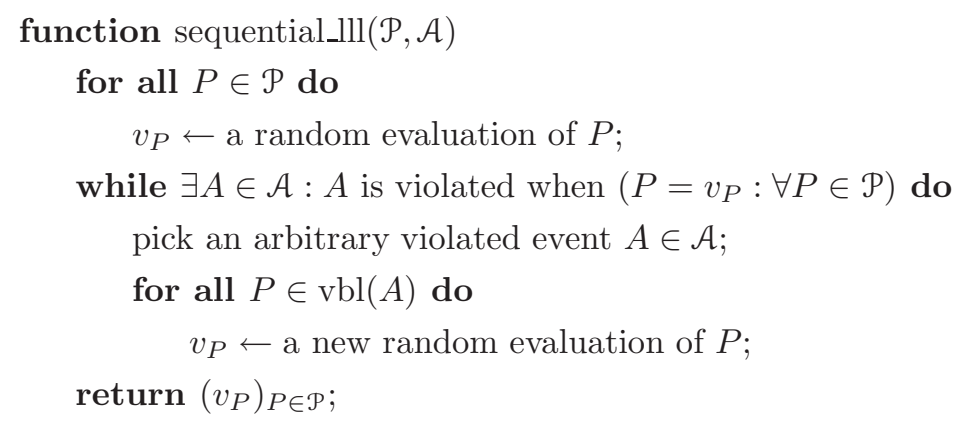

\section{Algorithm 1.1: the sequential solver}

The efficiency of the method clearly depends upon whether random values for each variable can be efficiently sampled and whether they can be efficiently checked against the given events. This is the case for almost all known applications of the lemma and it is less restrictive than previously known methods which required conditional probabilities or expectations to be computed. We will analyze the efficiency of the algorithm in terms of the expected number of times an event $A \in \mathcal{A}$ is resampled.

Theorem 1.2. Let $\mathcal{P}$ be a finite set of mutually independent random variables in a probability space. Let $\mathcal{A}$ be a finite set of events determined by these variables. If there exists an assignment of reals $x: \mathcal{A} \rightarrow(0,1)$ such that

$$
\forall A \in \mathcal{A}: \operatorname{Pr}[A] \leq x(A) \prod_{B \in \Gamma_{\mathcal{A}}(A)}(1-x(B))
$$

then there exists an assignment of values to the variables $\mathcal{P}$ not violating any of the events in $\mathcal{A}$. Moreover the randomized algorithm described above resamples an event $A \in \mathcal{A}$ at most an expected $x(A) /(1-x(A))$ times before it finds such an evaluation. Thus the expected total number of resampling steps is at most $\sum_{A \in \mathcal{A}} \frac{x(A)}{1-x(A)}$.

Our algorithm lends itself for parallelization. In the parallel version of the algorithm (Algorithm 1.2) we start again with the evaluation of the variables at a random point in the probability space, then in every step we select a maximal independent set $S$ in the subgraph of the dependency graph $G$ spanned by the violated events and resample all the variables these events depend on in parallel. That is, we take independent new samples of the variables in $\cup_{A \in S} \operatorname{vbl}(A)$ and keep the values assigned to the rest of the variables. We continue until we find an evaluation not violating any of the events. This algorithm can be considered a special case of the sequential algorithm, so the statement of Theorem 1.2 applies to the parallel version too. In order to give a logarithmic bound for the expected number of steps we assume slightly stronger bounds on the probabilities of the events.

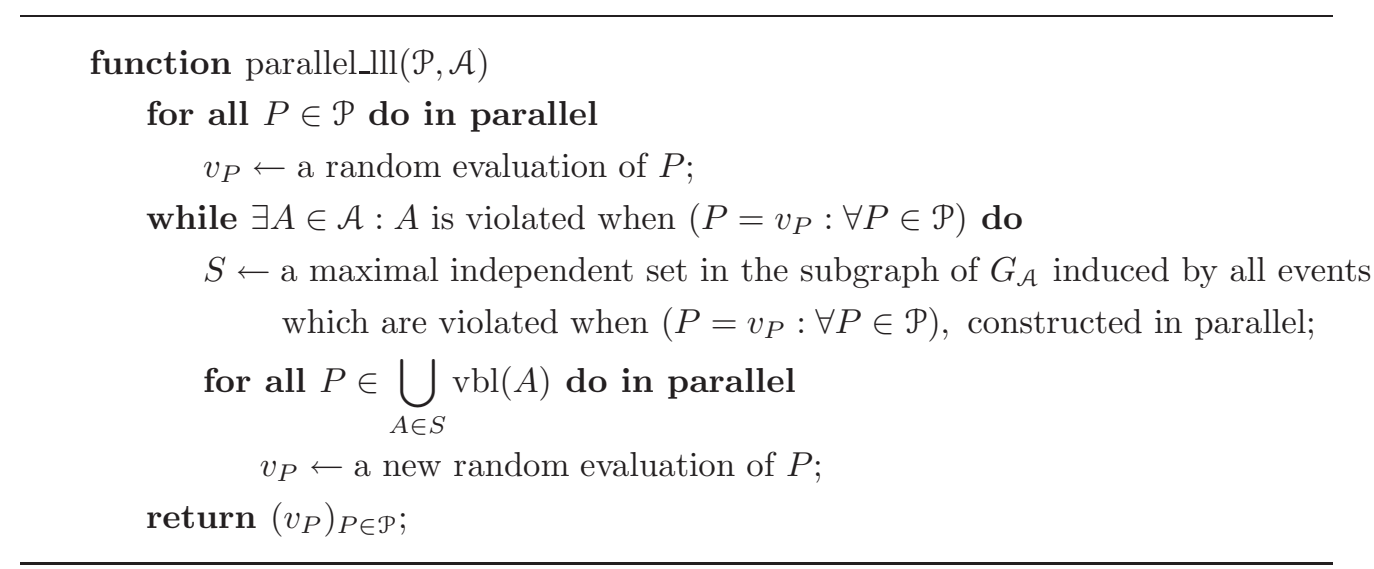

Algorithm 1.2: the parallel solver 
Theorem 1.3. Let $\mathcal{P}$ be a finite set of mutually independent random variables in a probability space. Let $\mathcal{A}$ be a finite set of events determined by these variables. If $\varepsilon>0$ and there exists an assignment of reals $x: \mathcal{A} \rightarrow(0,1)$ such that

$$
\forall A \in \mathcal{A}: \operatorname{Pr}[A] \leq(1-\varepsilon) x(A) \prod_{B \in \Gamma_{\mathcal{A}}(A)}(1-x(B))
$$

then the parallel version of our algorithm takes an expected $O\left(\frac{1}{\varepsilon} \log \sum_{A \in \mathcal{A}} \frac{x(A)}{1-x(A)}\right)$ steps before it finds an evaluation violating no event in $\mathcal{A}$.

There is not much about our algorithm that is inherently randomized. We can demonstrate that under additional conditions, most notably a constant bound on the maximum degree of the dependency graph, the same task can be performed by a deterministic procedure.

Theorem 1.4. Let $\mathcal{P}=\left\{P_{1}, P_{2}, \ldots, P_{n}\right\}$ be a finite set of mutually independent random variables in a probability space, each $P_{i}$ taking values from a finite domain $D_{i}$. Let $\mathcal{A}$ be a set of $m$ events determined by these variables. Consider the problem size to be $s:=m+n+\sum_{i=1}^{n}\left|D_{i}\right|$. Suppose there exists an algorithm that can compute, for each $A \in \mathcal{A}$ and each partial evaluation $\left(v_{i} \in D_{i}\right)_{i \in I}, I \subseteq[n]$ the conditional probability $\operatorname{Pr}\left[A \mid \forall i \in I: P_{i}=v_{i}\right]$ in time polynomial in s. Suppose, moreover, that the maximum degree of the dependency graph $G_{\mathcal{A}}$ is bounded by a constant, that is $\forall A \in \mathcal{A}:\left|\Gamma_{\mathcal{A}}(A)\right| \leq k$ for some constant $k$. If there is a constant $\varepsilon>0$ and an assignment of reals $x: \mathcal{A} \rightarrow(0,1)$ such that

$$
\forall A \in \mathcal{A}: \operatorname{Pr}[A] \leq(1-\varepsilon) x(A) \prod_{B \in \Gamma_{\mathcal{A}}(A)}(1-x(B))
$$

then a deterministic algorithm can find an evaluation of the variables such that no event occurs in time polynomial in $s$.

In Sections 2 and 3 we provide an analysis of the algorithm, proving Theorem 1.2, in Section 4 we analyze the parallel version and prove Theorem 1.3. In Section 5, we prove the correctness of the derandomized algorithm as claimed in Theorem 1.4. Section 6 contains a lopsided version of Theorem 1.2 and Section 7 has concluding remarks.

\section{Execution logs and witness trees}

Note that the decision which violated event $A \in \mathcal{A}$ to correct in each step can be taken completely arbitrarily. Let us fix any (deterministic or randomized) procedure for this selection, this makes the algorithm and the expected values we consider well defined. The selection method the algorithm uses does not matter for our analysis.

We need to record an accurate journal of what the algorithm does. Let $C: \mathbb{N} \rightarrow \mathcal{A}$ list the events as they have been selected for resampling in each step. If the algorithm terminates, $C$ is partial and defined only up to the given total number of steps carried out. We call $C$ the $\log$ of the execution. With a fixed selection discipline as described above, $C$ is now a random variable determined by the random choices the algorithm makes.

Recall that dependency graph $G$ is a graph on vertex set $\mathcal{A}$ where two distinct events $A, A^{\prime} \in \mathcal{A}$ are connected if $\operatorname{vbl}(A) \cap \operatorname{vbl}\left(A^{\prime}\right) \neq \emptyset$ and that $\Gamma(A)$ denotes the neighborhood of the vertex $A$ in $G$. We also use here the inclusive neighborhood $\Gamma^{+}(A):=\Gamma(A) \cup\{A\}$ of a vertex $A$.

A witness tree $\tau=\left(T, \sigma_{T}\right)$ is a finite rooted tree $T$ together with a labelling $\sigma_{T}: V(T) \rightarrow \mathcal{A}$ of its vertices with events such that the children of a vertex $u \in V(T)$ receive labels from $\Gamma^{+}\left(\sigma_{T}(u)\right)$. If distinct 
children of the same vertex always receive distinct labels we call the witness tree proper. To shorten notation, we will write $V(\tau):=V(T)$ and for any $v \in V(\tau)$, we write $[v]:=\sigma_{T}(v)$. Given the $\log C$, we will now associate with each resampling step $t$ carried out a witness tree $\tau_{C}(t)$ that can serve as a 'justification' for the necessity of that correction step. Let us define $\tau_{C}^{(t)}(t)$ to be an isolated root vertex labelled $C(t)$. Then going backwards through the $\log$, for each $i=t-1, t-2, \ldots, 1$, we distinguish two cases. If there is a vertex $v \in \tau_{C}^{(i+1)}(t)$ such that $C(i) \in \Gamma^{+}([v])$, then we choose among all such vertices the one having the maximum distance from the root and attach a new child vertex $u$ to $v$ that we label $C(i)$, thereby obtaining the tree $\tau_{C}^{(i)}(t)$. In the selection of the maximum distance vertex we break ties arbitrarily. If there is no vertex $v \in \tau_{C}^{(i+1)}(t)$ such that $C(i) \in \Gamma^{+}([v])$, then we skip time step $i$ and simply define $\tau_{C}^{(i)}(t):=\tau_{C}^{(i+1)}(t)$. Finally let $\tau_{C}(t):=\tau_{C}^{(1)}(t)$.

We say that the witness tree $\tau$ occurs in the $\log C$ if there exists $t \in \mathbb{N}$ such that $\tau_{C}(t)=\tau$.

Lemma 2.1. Let $\tau$ be a fixed witness tree and $C$ the (random) log produced by the algorithm.

(i) If $\tau$ occurs in $C$, then $\tau$ is proper.

(ii) The probability that $\tau$ appears in $C$ is at most $\prod_{v \in V(\tau)} \operatorname{Pr}[[v]]$.

Proof. Assume $\tau$ occurs in the $\log C$, so we have $\tau_{C}(t)=\tau$ for some $t \in \mathbb{N}$. For a vertex $v \in V(\tau)$ let $d(v)$ denote the depth of vertex $v$, that is its distance from the root and let $q(v)$ stand for the step of the algorithm constructing $\tau_{C}(t)$ in which $v$ was attached, that is, $q(v)$ is the largest value $q$ with $v$ contained in $\tau_{C}^{(q)}(t)$.

First note that if $q(u)<q(v)$ for vertices $u, v \in V(\tau)$ and $\operatorname{vbl}([u])$ and $\operatorname{vbl}([v])$ are not disjoint, then $d(u)>d(v)$. Indeed, when adding the vertex $u$ to $\tau_{C}^{(q(u)+1)}(t)$ we attach it to $v$ or to another vertex of equal or greater depth. As a consequence observe that for any two vertices $u, v \in V(\tau)$ at the same depth $d(v)=d(u),[u]$ and $[v]$ do not depend on any common variables, that is the labels in every level of $\tau$ form an independent set in $G$. In particular $\tau$ must be proper, establishing claim (i).

Consider the following procedure that we call $\tau$-check: In an order of decreasing depth (e.g., reversed breadth first search order) visit the vertices of $\tau$ and for a vertex $v$ take a random evaluation of the variables in $\operatorname{vbl}([v])$ (according to their distribution, independent of possible earlier evaluations) and check if the resulting evaluation violates $[v]$. We say that the $\tau$-check passes if all events were violated when checked.

Trivially, the $\tau$-check passes with probability exactly $\prod_{v \in V(\tau)} \operatorname{Pr}[[v]]$. The lemma follows from the observation that whenever $\tau$ occurs in the log and we run the $\tau$-check on the same random source it passes. For this coupling argument we have to specify explicitly how the algorithms use the random source. We assume that for each variable $P \in \mathcal{P}$ the random source produces an infinite list of independent random samples $P^{(0)}, P^{(1)}, \ldots$, and whenever either algorithm calls for a new random sample of $P$ we pick the next unused value from this sequence.

Having assumed that $\tau=\tau_{C}(t)$, we need to prove that the $\tau$-check passes, that is, when it considers a vertex $v \in V(\tau)$ and takes random samples of the variables in $\operatorname{vbl}([v])$, the resulting evaluation violates $[v]$. Let us fix the vertex $v$ and for $P \in \operatorname{vbl}([v])$ let $S(P)$ be the set of vertices $w \in V(\tau)$ with $d(w)>d(v)$ and $P \in \operatorname{vbl}([w])$. When the $\tau$-check considers the vertex $v$ and samples $P$ the random source gives $P^{(|S(P)|)}$. This is because the $\tau$-check visits the vertices in order of decreasing depth and among the vertices with depth equal to $d(v)$ only the label of $v$ depends on $P$, so before the $\tau$-check considers $v$ it had sampled $P$ exactly when it was considering the vertices in $S(P)$.

In step $q(v)$, our algorithm chooses the event $[v]$ for resampling, so $[v]$ must be violated before this resampling. We claim that for $P \in \operatorname{vbl}([v])$ the current value of the variable $P$ is $P^{(|S(P)|)}$ at this time. Indeed, $P$ was sampled at the beginning of the algorithm and then at the steps $q(w)<q(v)$ for $w \in S(P)$. As the $\tau$-check has these exact same values for the variables in $\operatorname{vbl}([v])$ when considering $v$ it also must find that $[v]$ is violated, proving (ii). 
For any event $A \in \mathcal{A}$, let us denote by $N_{A}$ the random variable that counts how many times the event $A$ is resampled during the execution of our algorithm. If $C$ is the $\log$ of the execution of our algorithm, then $N_{A}$ is the number of occurrences of $A$ in this log and also the number of distinct proper witness trees occurring in $C$ that have their root labeled $A$. The latter statement holds because if $t_{i}$ is the $i$-th time step with $C\left(t_{i}\right)=A$, then obviously the tree $\tau_{C}\left(t_{i}\right)$ contains exactly $i$ vertices labelled $A$, thus $\tau_{C}\left(t_{i}\right) \neq \tau_{C}\left(t_{j}\right)$ unless $i=j$. Therefore one can bound the expectation of $N_{A}$ simply by summing the bounds in Lemma 2.1 on the probabilities of the occurrences of the different proper witness trees. In the next section we do just that by relating these probabilities to a random process.

\section{Random generation of witness trees}

Let us fix an event $A \in \mathcal{A}$ and consider the following multitype Galton-Watson branching process for generating a proper witness tree having its root labelled $A$. In the first round, we produce a singleton vertex labelled $A$. Then in each subsequent round, we consider each vertex $v$ produced in the previous round independently and, again independently, for each event $B \in \Gamma^{+}([v])$ identical or adjacent to $[v]$ in the dependency graph, we add to $v$ a child node carrying the label $B$ with probability $x(B)$ or skip that label with probability $1-x(B)$. All these choices are independent. The process continues until it dies out naturally because no new vertices are born in some round (depending on the probabilities used, there is, of course, the possibility that this never happens).

Let $x^{\prime}(B):=x(B) \prod_{C \in \Gamma(B)}(1-x(C))$. For the probability that the described Galton-Watson process yields a prescribed proper witness tree we obtain the following.

Lemma 3.1. Let $\tau$ a fixed proper witness tree with its root vertex labelled $A$. The probability $p_{\tau}$ that the Galton-Watson process described above yields exactly the tree $\tau$ is

$$
p_{\tau}=\frac{1-x(A)}{x(A)} \prod_{v \in V(\tau)} x^{\prime}([v])
$$

Proof. For a vertex $v \in V(\tau)$ we denote by $W_{v} \subseteq \Gamma^{+}([v])$ the set of inclusive neighbors of $[v]$ that do not occur as a label of some child node of $v$. Then clearly, the probability that the Galton-Watson process produces exactly $\tau$ is given by

$$
p_{\tau}=\frac{1}{x(A)} \prod_{v \in V(\tau)}\left(x([v]) \prod_{u \in W_{v}}(1-x([u]))\right),
$$

where the leading factor accounts for the fact the the root is always born. In order to get rid of the $W_{v}$, we can rewrite this expression in an obvious way to obtain

$$
p_{\tau}=\frac{1-x(A)}{x(A)} \prod_{v \in V(\tau)}\left(\frac{x([v])}{1-x([v])} \prod_{u \in \Gamma^{+}([v])}(1-x([u]))\right),
$$

where again we have to account for the root separately. Replacing inclusive by exclusive neighborhoods, this simplifies to

$$
p_{\tau}=\frac{1-x(A)}{x(A)} \prod_{v \in V(\tau)}\left(x([v]) \prod_{u \in \Gamma([v])}(1-x([u]))\right)=\frac{1-x(A)}{x(A)} \prod_{v \in V(\tau)} x^{\prime}([v]) .
$$


Let $\mathcal{T}_{A}$ denote the set of all proper witness trees having the root labelled $A$. We have

$$
\mathbb{E}\left(N_{A}\right)=\sum_{\tau \in \mathcal{T}_{A}} \operatorname{Pr}[\tau \text { appears in the } \log C] \leq \sum_{\tau \in \mathcal{T}_{A}} \prod_{v \in V(\tau)} \operatorname{Pr}[[v]] \leq \sum_{\tau \in \mathcal{T}_{A}} \prod_{v \in V(\tau)} x^{\prime}([v]),
$$

where the first inequality follows from Lemma 2.1, while the second follows from the assumption in Theorem 1.2, We further have

$$
\mathbb{E}\left(N_{A}\right) \leq \sum_{\tau \in \mathcal{T}_{A}} \prod_{v \in V(\tau)} x^{\prime}([v])=\frac{x(A)}{1-x(A)} \sum_{\tau \in \mathcal{T}_{A}} p_{\tau} \leq \frac{x(A)}{1-x(A)},
$$

where the equality comes from Lemma 3.1, while the last inequality follows from the fact that the GaltonWatson process produces exactly one tree at a time (not necessarily one from $\mathcal{T}_{A}$ since it might also grow infinite). This concludes the proof of Theorem 1.2 .

\section{Analyzing the parallel algorithm}

Let us consider an arbitrary execution of the parallel version of the algorithm. We choose an arbitrary ordering of the violated events selected for resampling at each step and consider that these resamplings are done in that order sequentially. This way we obtain an execution of the sequential algorithm. Let $S_{j}$ be the segment of the $\log C$ of this execution that corresponds to resamplings done in step $j$ of the parallel algorithm. We call the maximal depth of a vertex in a witness tree the depth of the tree.

Lemma 4.1. If $t \in S_{j}$, then the depth of $\tau_{C}(t)$ is $j-1$.

Proof. Let $t_{k}$ be the first number in the segment $S_{k}$ and let $\tau_{k}=\tau_{C}^{\left(t_{k}\right)}(t)$ for $k \leq j$. As the events resampled in the $j$-th parallel step are independent, the root is the only vertex of $\tau_{j}$. For $k<j$ we obtain $\tau_{k}$ from $\tau_{k+1}$ by attaching some vertices corresponding to the $k$-th parallel step of the algorithm. As these vertices have independent labels they can only add one to the depth. To see that they do add one to the depth consider a vertex $v$ of $\tau_{k+1}$ of maximal depth. This vertex corresponds to a resampling of the event $[v]$ some time after step $k$ of the parallel algorithm. If $\tau_{k}$ has no vertex with higher depth than $v$, then from the parallel step $k$ to the resampling corresponding to $v$ no event from $\Gamma^{+}([v])$ was resampled. But this implies that $[v]$ was already violated at parallel step $k$ and we did not select a maximal independent set of violated events there for resampling. The contradiction shows that the depth of $\tau_{k}$ is indeed one more than that of $\tau_{k+1}$. To finish the proof notice that $\tau_{C}(t)=\tau_{1}$.

Let $Q(k)$ denote the probability, that the parallel algorithm makes at least $k$ steps. By Lemma 4.1 some witness tree of depth $k-1$ must occur in the log in this case. Notice that a depth $k-1$ witness tree has at least $k$ vertices. Let $\mathcal{T}_{A}(k)$ be the set of witness trees in $\mathcal{T}_{A}$ having at least $k$ vertices. We have

$Q(k) \leq \sum_{A \in \mathcal{A}} \sum_{\tau \in \mathcal{T}_{A}(k)} \operatorname{Pr}[\tau$ appears in the $\log C] \leq \sum_{A \in \mathcal{A}} \sum_{\tau \in \mathcal{T}_{A}(k)} \prod_{v \in V(\tau)} \operatorname{Pr}[[v]] \leq(1-\varepsilon)^{k} \sum_{A \in \mathcal{A}} \sum_{\tau \in \mathcal{T}_{A}} \prod_{v \in V(\tau)} x^{\prime}([v])$

where the last inequality follows from the assumption in Theorem 1.3 . Then, as before, we have

$$
Q(k) \leq(1-\varepsilon)^{k} \sum_{A \in \mathcal{A}} \sum_{\tau \in \mathcal{T}_{A}} \prod_{v \in V(\tau)} x^{\prime}([v])=(1-\varepsilon)^{k} \sum_{A \in \mathcal{A}} \frac{x(A)}{1-x(A)} \sum_{\tau \in \mathcal{T}_{A}(k)} p_{\tau} \leq(1-\varepsilon)^{k} \sum_{A \in \mathcal{A}} \frac{x(A)}{1-x(A)} .
$$

This bound easily implies Theorem 1.3 . 


\section{A deterministic variant}

It is possible to derandomize our algorithm under the additional assumptions listed in Theorem 1.4, The idea is to create a list of all large potential witness trees which, if done with care, remains polynomial in length, and then to search for a table of evaluations of all random variables which, substituted for the random source in the original randomized variant, guarantees that the running time will be low.

To begin with, let us note that we can assume the weights $x(A)$ for $A \in \mathcal{A}$ to be bounded away from 1. If they are not, we can simply replace $x(A)$ by, say, $\tilde{x}(A):=(1-\epsilon / 2) x(A)$ for all $A$ and $\epsilon$ by $\epsilon / 2$. It is easily checked that all requirements are still satisfied and now the weights $\tilde{x}(A)$ are upper bounded by $(1-\epsilon / 2)$.

Let $\left(v_{i}^{(j)} \in D_{i}\right)_{1 \leq i \leq n, j \in \mathbb{N}}$ be sequences of values for the variables. Suppose we replace the random source by these sequences in such a way that we take $v_{i}^{(j)}$ as the $j$-th sample $P_{i}^{(j)}$ and now run either the parallel or the sequential algorithm as detailed in the previous sections. Recalling the proof of Lemma 2.1, let us say that a witness tree $\tau$ is consistent with the sequences $\left(v_{i}^{(j)} \in D_{i}\right)_{1 \leq i \leq n, j \in \mathbb{N}}$ if the $\tau$-check passes when substituting them for the random source. We have proved that if the values $v_{i}^{(j)}$ are selected at random, then the expected number of witness trees which are consistent with them and which feature at least $k$ vertices is bounded by $(1-\varepsilon)^{k} \sum_{A \in \mathcal{A}} \frac{x(A)}{1-x(A)}$ and if the weights are bounded away from 1 , this is in turn bounded by $\mathcal{O}\left(m(1-\varepsilon)^{k}\right)$. There exists therefore a constant $c$ such that the expected number of witness trees of size at least $c \log m$ that are consistent with the sequences is at most $1 / 2$. With probability at least $1 / 2$, no consistent witness tree of size at least $c \log m$ exists at all. Since no variable can then be reassigned more than $c \log m$ times, we can restrict ourselves to finding values $\left(v_{i}^{(j)} \in D_{i}\right)_{1 \leq i \leq n, 0 \leq j \leq c \log m}$ such that no large tree becomes consistent. Such values can be found sequentially using the method of conditional expectations. First of all, we have to make sure that the number of witness trees we have to consider is not too large, which is demonstrated by the following lemma.

Lemma 5.1. Suppose the maximum degree of the dependency graph is bounded by $k$ as required in the theorem. Let $u \in \mathbb{N}$. If there exists a witness tree of size at least $u$ that is consistent with given evaluation sequences $\left(v_{i}^{(j)}\right)_{1 \leq i \leq n, j \in \mathbb{N}}$, then there also exists a witness tree of some size in the range $[u,(k+1) u]$ that is also consistent with the sequences.

Proof. To arrive at a contradiction, assume that the claim is wrong for some value $u$. Then let $\tau$ be a smallest counterexample, that is a consistent witness tree of size larger than $(k+1) u$ such that there exists no consistent witness tree of a size in the range $[u,(k+1) u]$. Due to the bounded maximum degrees of the dependency graph, each node in $\tau$ has at most $k+1$ children. Let $w_{1}, w_{2}, \ldots, w_{j}$ with $j \leq k+1$ be the immediate children of the root. Now we can build $j$ distinct witness trees that are all consistent with the sequences as follows: traverse $\tau$ in the usual level-by-level fashion bottom-up, starting at the lowest level and ending at the root. Consider this sequence of corrections to be a $\log C^{\prime}$ of length $|\tau|$ and now construct for the $j$ penultimate correction steps in $C^{\prime}$ (which obviously correspond to $w_{1}$ through $w_{j}$ ) the corresponding witness trees $\tau_{i}:=\tau_{C^{\prime}}(|\tau|-i)$ for $i=1,2, \ldots, j$. For obvious reasons, each $\tau_{i}$ is consistent with the sequences. Moreover, since $\tau_{i}$ must contain at least as many vertices as the subtree of $\tau$ rooted at $w_{i}$, there must be at least one $i$ such that $\tau_{i}$ has at least $(|\tau|-1) /(k+1)$ vertices. Since $\tau$ has at least $(k+1) u+1$ vertices, $\tau_{i}$ has at least $u$ of them, implying that $\tau_{i}$ either contradicts the assumption or constitutes a smaller counterexample.

A deterministic algorithm can now proceed as follows. Enumerate all witness trees that have a size in the range $[c \log m,(k+1) c \log m]$ in a list $L$. There is clearly a polynomial number of those given that the dependency graph has bounded degrees. Now, in any ordering, go through all index pairs $(i, j)$ with $1 \leq i \leq n, 0 \leq j \leq c \log m$, computing suitable values for $v_{i}^{(j)}$ incrementally. For each of them, consider each possible value of $D_{i}$ that we could assign to $v_{i}^{(j)}$ and then compute the expected number of trees in $L$ 
that become consistent with the sequences of values given that all values chosen so far and $v_{i}^{(j)}$ are fixed and the yet uncomputed values are considered to be random. In the beginning when no value is fixed so far, that expected value is at most $1 / 2$. Clearly, in each step we can preserve that it remains at most $1 / 2$ by selecting a value for $v_{i}^{(j)}$ that minimizes that expectation. Once all values are fixed, the expectation has to coincide with the actual number of consistent witness trees in $L$ and therefore that number has to be zero.

Computing the probability of each tree $\tau \in L$ given that some evaluations are random and others are fixed can be done easily by traversing $\tau$ in the usual bottom-up level-by-level fashion (note that each time we encounter a variable during this traversal we increment a counter pointing to the sample that has to be used as the current value for this variable), for each vertex computing the conditional probability that the corresponding event is violated given the values for the samples fixed so far (using the algorithm we assume to exist in the hypothesis) and multiplying those probabilities.

After this polynomial preprocessing step, we run the usual algorithm substituting our tailored values for the random source having guaranteed that it will terminate after a polynomial number of steps.

\section{$6 \quad$ The Lopsided Local Lemma}

In this section we study a lopsided version of the Local Lemma that is slightly outside the framework of Theorem 1.1. Using lopsided dependence, which is an adaptation of the notion in ES91 to our setting, we formulate our main result in a slightly more general form. We started with the original formulation of Theorem 1.2 because we find it more intuitive and it is general enough for most applications of the Local Lemma. This lopsided generalization can also be applied to the derandomized variant of Theorem 1.4. On the other hand, we could not find an effective parallelization.

We are still in the setting of Theorem 1.2: $\mathcal{P}$ is a finite set of mutually independent random variables in a probability space and $\mathcal{A}$ is a finite set of events determined by these variables. We say that two events $A, B \in \mathcal{A}$ are lopsidependent if there exist two evaluations $f$ and $g$ of the variables in $\mathcal{P}$ that differ only on variables in $\operatorname{vbl}(A) \cap \operatorname{vbl}(B)$ such that $f$ violates $A$ and $g$ violates $B$ but either $f$ does not violate $B$ or $g$ does not violate $A$. The lopsidependency graph is the graph on the vertex set $\mathcal{A}$, where lopsidependent events are connected by an edge. We write $\Gamma^{\prime}(A)=\Gamma_{\mathcal{A}}^{\prime}(A)$ for the neighborhood of an event $A$ in this graph.

Clearly, if $\operatorname{vbl}(A)$ is disjoint from $\operatorname{vbl}(B)$, then $A$ and $B$ cannot be lopsidependent, so we have $\Gamma^{\prime}(A) \subseteq$ $\Gamma(A)$. Substituting $\Gamma^{\prime}(A)$ for $\Gamma(A)$ in the statement of Theorem 1.2 makes the assumption weaker and therefore the theorem itself stronger.

We call an event $A \in \mathcal{A}$ elementary, if there is a single evaluation of the variables in $\operatorname{vbl}(A)$ violating $A$. Lopsidependence between elementary events is more intuitive: the elementary events $A$ and $B$ are lopsidependent if they are disjoint (mutually exclusive). Elementary events form a very special case of events considered in the Local Lemma, but in case the variables in $\operatorname{vbl}(A)$ have finite domain, any event $A$ is the union of finitely many elementary events determined by the same set of variables. Avoiding $A$ is equivalent to avoiding all of these elementary events and such a "breaking up" of event $A$ is not too costly in the following sense. If the assignment $x: \mathcal{A} \rightarrow(0,1)$ satisfies the (slightly stronger than usual) condition

$$
\forall A \in \mathcal{A}: \operatorname{Pr}[A] \leq x(A) \prod_{B \in \Gamma^{+}(A)}(1-x(B)),
$$

then we can break up all the events $A \in \mathcal{A}$ into elementary events and find a corresponding assignment to the elementary events satisfying the same condition. Even in cases where a suitable assignment is not possible for the set $\mathcal{A}$, breaking up the events may cause many of the dependencies among the elementary events to disappear when considering lopsidependence, and Theorem 6.1 may become applicable. 
An important application where using lopsidependence has proven to be effective is the satisfiability problem. In a CNF formula, two clauses $A$ and $B$ are said to have a conflict if one contains a literal $l$ and the other one its complement $\bar{l}$. When conducting the random experiment of sampling a truth assignment to each variable independently, the (elementary) events that $A$ is violated or that $B$ is violated, respectively, are lopsidependent on one another. If, on the other hand, $A$ and $B$ merely overlap in some literal, then correcting either of them in the case of being violated will not harm the other one. Hence, being in conflict is the more relevant notion to analyze dependencies in a SAT problem. The lopsided Local Lemma has been effectively used, e.g. by Berman, Karpinski and Scott in [BKS03, to prove better bounds on the number of occurrences per variable that can be allowed while guaranteeing a satisfying assignment. Proofs of this type can be made constructive using the method we present here.

Theorem 6.1. Let $\mathcal{P}$ be a finite set of mutually independent random variables in a probability space. Let $\mathcal{A}$ be a finite set of events determined by these variables. If there exists an assignment of reals $x: \mathcal{A} \rightarrow(0,1)$ such that

$$
\forall A \in \mathcal{A}: \operatorname{Pr}[A] \leq x(A) \prod_{B \in \Gamma_{\mathcal{A}}^{\prime}(A)}(1-x(B))
$$

then there exists an assignment of values to the variables $\mathcal{P}$ not violating any of the events in $\mathcal{A}$. Moreover our randomized algorithm resamples an event $A \in \mathcal{A}$ at most an expected $x(A) /(1-x(A))$ times before it finds such an evaluation. Thus the expected total number of resampling steps is at most $\sum_{A \in \mathcal{A}} \frac{x(A)}{1-x(A)}$.

As the proof of this theorem is almost identical to that of Theorem 1.2 we concentrate on the few differences. We define $\Gamma^{\prime+}(A)=\Gamma_{\mathcal{A}}^{\prime+}(A)=\Gamma_{\mathcal{A}}^{\prime}(A) \cup\{A\}$ for $A \in \mathcal{A}$. Given a $\log C$ of an execution of Algorithm 1.1 and a time index $t$, we define the lopsided witness tree $\varrho_{C}(t)$ similarly to the usual witness tree $\tau_{C}(t)$, but now the children of a vertex labelled $A$ will be labelled from $\Gamma^{\prime+}(A)$. Formally we start with the root labeled $C(t)$ and going through the time steps $i=t-1, t-2, \ldots, 1$ of the log we add a new vertex labelled $C(i)$ every time $C(i) \in \Gamma^{+}(A)$ for a label $A$ of a vertex of the tree constructed so far. If such a vertex exists we choose one as far from the root as possible and attach the new vertex of label $C(i)$ to this existing vertex.

We denote the label of a vertex $v$ by $[v]$ and call a witness tree a proper lopsided witness tree if the children of any vertex $v$ receive distinct labels from $\Gamma^{\prime+}([v])$. Clearly, $\varrho_{C}(t)$ is a proper lopsided witness tree, but the equivalent of part (ii) of Lemma 2.1 is less trivial. We state and prove it below. One also has to modify the Galton-Watson branching process considered to produce a proper lopsided witness tree. With these modifications the original proof of Theorem 1.2 carries over to the lopsided version.

Lemma 6.2. Let $\tau$ be a fixed proper lopsided witness tree and $C$ the (random) log produced by the algorithm. The probability that $\tau=\varrho_{C}(t)$ holds for some $t$ is at most $\prod_{v \in V(\tau)} \operatorname{Pr}[[v]]$.

Proof. We prove this statement with the same coupling argument as in Lemma 2.1 if $\tau=\varrho_{C}(t)$, then the $\tau$-check (as defined in the proof of Lemma 2.1) passes if both the algorithm and the $\tau$-check use the same list of independent random samples $P^{(0)}, P^{(1)}, \ldots$ for the variables $P \in \mathcal{P}$. Recall that the $\tau$-check considers the vertices of $\tau$ in some fixed order $v_{1}, \ldots, v_{s}$ of non-increasing distance from the root and, when considering $v_{i}$ draws new samples for the variables in vbl $\left(\left[v_{i}\right]\right)$ and checks if they violate $\left[v_{i}\right]$. Clearly, the probability that the $\tau$-check passes (meaning that each event is violated when checked) is exactly $\prod_{v \in V(\tau)} \operatorname{Pr}[[v]]$.

Let us fix the sequences $P^{(0)}, P^{(1)}, \ldots$ of random samples of the variables $P \in \mathcal{P}$. The $\log$ of our algorithm is still not determined because of the arbitrary choices we make in selecting the violated event to resample. If a possible $\log C$ satisfies $\varrho_{C}(t)=\tau$ for some $t$ we denote by $q_{C}(v)$ the time step the vertex $v$ was added during the construction of $\varrho_{C}(t)$. In particular we have $q_{C}(r)=t$ for the root $r$ of $\tau$ and we have $C\left(q_{C}(v)\right)=[v]$. If there are several possible $\operatorname{logs} C$ with $\tau=\varrho_{C}(t)$ for some $t$, then we choose the one that minimizes $w(C):=\sum_{i=1}^{s}(s+1-i) q_{C}\left(v_{i}\right)$. We claim that with this choice we have $q_{C}\left(v_{i}\right)=i$ for 
$i=1, \ldots, s$. Assuming the claim it is clear that the $\tau$-check passes since when it considers $v_{i}$ it will draw the same random values for the variables in $\operatorname{vbl}\left(\left[v_{i}\right]\right)$ that makes the selection of $C(i)=\left[v_{i}\right]$ a valid choice for resampling in the $i$ th step of our algorithm.

To prove the claim above notice first that the definition of lopsidependence ensures that if $C$ is a possible $\log$ of our algorithm and for some $j$ the events $C(j)$ and $C(j+1)$ are not lopsidependent, then the $\log C^{\prime}$ obtained from $C$ by reversing the order of these two resamplings is still possible. Furthermore if $\tau$ can be obtained as $\varrho_{C}(t)$ for some $t$ then $\tau$ can also be obtained as $\varrho_{C^{\prime}}(t)$ for some $t$. Now assume that the claim does not hold. In this case there must exist indices $i$ and $j$ with $q_{C}\left(v_{i}\right)=j+1$ and $q_{C}\left(v_{i^{\prime}}\right) \neq j$ for any $i^{\prime}<i$. But this means that during the construction of $\tau=\varrho_{C}(t)$ when considering time step $j$ we did not attach a new vertex labeled $C(j)$, or if we did, it went on the level of $v_{i}$ or even higher. In either case $C(j)$ must not be lopsidependent from $C(j+1)=\left[v_{i}\right]$, neither can $C(j)$ and $C(j+1)$ coincide. Thus the $\log C^{\prime}$ obtained from $C$ by switching $C(j)$ and $C(j+1)$ is also a possible log producing $\tau$ as a lopsided witness tree, and we also have $w\left(C^{\prime}\right)<w(C)$, a contradiction.

\section{Conclusion}

The bound $x(A) /(1-x(A))$ in Theorem 1.2 is tight, but it is only achieved in the rather uninteresting case, when $A$ is an isolated vertex of the dependency graph and we set $x(A)=\operatorname{Pr}[A]$. Consequently the bound on the total number of resampling steps is achieved only if all the events in $\mathcal{A}$ are independent. To see that the expected number of resamplings of an event $A \in \mathcal{A}$ cannot achieve the bound $x(A) /(1-x(A))$ unless $A$ is isolated in the dependency graph notice that for equality any proper witness tree with the root labelled $A$ must be present in the log with positive probability, but only those can be present where the labels on each level form an independent set in the dependency graph.

To implement the sequential version of our algorithm we need only to assume that we have an algorithm sampling the variables in $\mathcal{P}$ and another that finds the set of violated events for a given evaluation of the variables. For the parallel version we also need to be able to find a maximal independent set of violated vertices of the dependence graph. Luby's randomized algorithm finds a maximal independent set in any graph in logarithmic expected time using a processor associated with each vertex of the graph. As we have to find an expected logarithmic number of maximal independent sets, one after the other, the total running time of this part of the algorithm is $O\left(\log ^{2} m\right)$, where $m=|V(G)|=|\mathcal{A}|$. Sampling and finding the violated indices is typically faster, so this might be the bottleneck of our algorithm. One can reduce the time required to find an independent set to a constant by implementing only a single step of Luby's algorithm. The result will not be a maximal independent set, nevertheless we can use it to perform the corresponding resamplings in parallel, but the expected number of steps this version of the algorithm takes is yet to be analyzed.

Finally, it remains an interesting open question whether it is possible to derandomize our algorithm even if the degrees of the dependency graph are unbounded.

\section{Acknowledgements}

Many thanks go to Dominik Scheder and Philipp Zumstein for various very helpful comments and to Emo Welzl for many fruitful discussions and the continuous support. 


\section{References}

[Knu69] Donald E. Knuth. The Art of Computer Programming, Vol. I, Addison Wesley, London, 1969, p. 396 (Exercise 11).

[EL75] Paul Erdős and László Lovász. Problems and results on 3-chromatic hypergraphs and some related questions. In A. Hajnal, R. Rado and V.T. Sós, editors, Infinite and Finite Sets (Colloq., Keszthely, 1973; dedicated to P. Erdős on his 60th birthday), volume II, pages 609-627. North-Holland, 1975.

[ES91] Paul Erdős and Joel Spencer. Lopsided Lovász Local Lemma and Latin Transversals. Discrete Applied Mathematics, 30:151-154, 1991.

[Bec91] Jószef Beck. An Algorithmic Approach to the Lovász Local Lemma. Random Structures and Algorithms, 2(4):343-365, 1991.

[Alo91] Noga Alon. A parallel algorithmic version of the local lemma. Random Structures and Algorithms, 2(4):367-378, 1991.

[KST93] Jan Kratochvíl and Petr Savický and Zsolt Tuza. One more occurrence of variables makes satisfiability jump from trivial to NP-complete. SIAM J. Comput., Vol. 22, No. 1, pp. 203-210, 1993.

[MR98] Michael Molloy and Bruce Reed. Further Algorithmic Aspects of the Local Lemma. In Proceedings of the 30th Annual ACM Symposium on the Theory of Computing, pages 524-529, 1998.

[CS00] Artur Czumaj and Christian Scheideler. Coloring non-uniform hypergraphs: a new algorithmic approach to the general Lovász local lemma. Symposium on Discrete Algorithms, 30-39, 2000.

[BKS03] Piotr Berman, Marek Karpinski, and Alexander D. Scott. Approximation hardness and satisfiability of bounded occurrence instances of SAT. Electronic Colloquium on Computational Complexity (ECCC), 10(022), 2003.

[Mos06] Robin A. Moser. On the Search for Solutions to Bounded Occurrence Instances of SAT. Not published. Semester Thesis, ETH Zürich. 2006.

[PT09] János Pach and Gábor Tardos. Conflict-free colorings of graphs and hypergraphs. Manuscript, 2009.

[Sri08] Aravind Srinivasan. Improved algorithmic versions of the Lovász Local Lemma. Proceedings of the nineteenth annual ACM-SIAM symposium on Discrete algorithms (SODA), San Francisco, California, pp. 611-620, 2008.

[Wel08] Emo Welzl. Boolean Satisfiability - Combinatorics and Algorithms. Lecture notes, Version Fall 2008.

[Mos08] Robin A. Moser. Derandomizing the Lovász Local Lemma more Effectively. Eprint, arXiv:0807.2120v2, 2008 .

[Mos09] Robin A. Moser. A constructive proof of the Lovász Local Lemma. Proceedings of the 41st annual ACM Symposium on Theory of Computing (STOC 2009), to appear. Available at arXiv:0810.4812v2, 2008. 